\title{
Numerical suppression of the twin-image in in-line holography of a volume of micro-objects
}

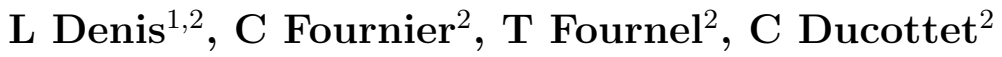 \\ ${ }^{1}$ Ecole Supérieure de Chimie Physique Electronique de Lyon, 43 bd du 11 Novembre \\ 1918, F-69616 Villeurbanne, France \\ ${ }^{2}$ Laboratoire Hubert Curien (ex-LTSI) ; CNRS, UMR5516 ; Université Jean Monnet; \\ 18 rue Pr Benoît Lauras, F-42000 Saint-Etienne, France \\ E-mail: loic.denis@cpe.fr \\ PACS numbers: 07.05.P, 42.40
}

Keywords: digital holography; in-line holography; deconvolution; twin-image.

Submitted to: Meas. Sci. Technol.

\begin{abstract}
We address the twin-image problem that arises in holography due to the lack of phase information in intensity measurements. This problem is of great importance in in-line holography where spatial elimination of the twin-image cannot be carried out as in off-axis holography.

A unifying description of existing digital suppression methods is given in the light of deconvolution techniques. Holograms of objects spread in 3D cannot be processed through available approaches. We suggest an iterative algorithm and demonstrate its efficacy on both simulated and real data. This method is suitable to enhance the reconstructed images from a digital hologram of small objects.
\end{abstract}




\section{Introduction}

Twin (i.e. virtual) images were considered as a limitation of holography since its very beginnings[1]. They are defocused images of the holographed objects that superimpose on the real images of the objects in the optical or numerical reconstructions. Their origin is tied to the principle of in-line holography and is recalled at the end of this introductory section.

The twin-image problem has received considerable attention since Gabor's pioneering work on holography. It is still a concern today as the in-line holographic setup is widely used in optical holography, soft X-rays holography and electronic microscopy. Basically, two classes of approaches have been proposed to tackle this problem: a first class which involves a change in the hologram-recording setup, and a second class that attempt to numerically suppress the twin-image. The best known method in the first category is off-axis holography, first suggested by Leith and Upatnieks[1]. An offaxis hologram leads to spatially separated real and twin images at the reconstruction step. The real image can then be selected by spatially filtering the reconstructed planes[2]. The digital off-axis setup however proves less relevant for the study of a collection of small objects due to sampling constraints[3] and practical considerations (robustness to vibrations). In-line digital holography is therefore preferred in this context and the twin-image problem has still to be addressed. By combining different holograms of the same objects recorded at distances with $\lambda / 4$ shifts, the phase shifting technique[4] performs phase reconstruction and therefore achieves twin-image suppression. Optical scanning holography[5] also reconstructs the phase thanks to an additional modulation/demodulation step. These two techniques are however limited to non-moving objects since the shifts or scans must be performed while the objects remain fixed. They are therefore irrelevant for the study of objects in motion. In this paper, we address the techniques adapted to the study of micro-objects in a volume. We consider only the in-line Gabor setup in the following analysis.

Numerical techniques (i.e. methods of the second aforementioned category) attempt to improve the system response $R$ of the holographic imaging technique (hologram recording + reconstruction) by changing the reconstruction step. Many techniques have been described in the literature as following a different approach. We provide in this paper, a unifying description of some of them in the light of deconvolution techniques in section 2. We show that the case of objects spread in a volume requires the development of a specific algorithm. Section 3 describes the suggested algorithm as an extension of existing iterative techniques. We show results on simulated and real data and discuss the applications and limits of the algorithm in section 4. Before describing twin-image suppression methods, we recall the origin of the twin-image phenomenon and give a quantitative description of twin-images.

Twin-image origin and characteristics: Hologram formation corresponds to the recording of the intensity pattern of the free propagated waves diffracted by the objects. 
Hologram reconstruction however does not exactly solves the inverse propagation problem but rather propagates further the intensity pattern. The complete system response $R$ of a point object in the focus plane therefore is not punctual but is the sum of a (real) punctual response and a (twin) defocused pattern $[6]^{*}$ :

$$
R(x, y)=\delta(x, y)+2 \Re\left[\underline{h_{2 z}}(x, y)\right],
$$

with $\delta(x, y)$ denoting Dirac's distribution, $\Re$ the real part, and $z$ the distance separating the point object and the hologram. The symbol $\underline{h_{z}}$ is Fresnel function of parameter $z$ and is the convolution kernel that models, under Fresnel's approximation, the diffraction at distance $z$. It is defined by:

$$
\underline{h_{z}}(x, y)=\frac{1}{\underline{j} \lambda z} \exp \left(\underline{j} \pi \frac{x^{2}+y^{2}}{\lambda z}\right) .
$$

The symbols $x$ and $y$ are the spatial coordinates in transverse planes (i.e. orthogonal to the optical axis) and $z$ is the depth coordinate (along the optical axis). The symbol $\lambda$ is the radiation wavelength and $j=\sqrt{-1}$. By convention, we underline complex-valued variables in this paper.

It is worth noting that a point object is recorded, in the hologram plane, as an intensity pattern which is classically approximated by the difference: $1-2 \Re\left(\underline{h_{z}}\right)[6]$. This difference can be expanded into three terms: $1-2 \Re\left(\underline{h_{z}}\right)=1-\underline{h_{z}}-\underline{h_{-z}}$ due to the conjugation property $\operatorname{conj}\left(\underline{h_{z}}\right)=h_{-z}$. The hologram intensity can then be seen as the diffraction amplitude created by two symmetrical objects (resp. real and virtual) located on both sides of the hologram, hence the twin-image can be interpreted as the diffraction pattern of a virtual object.

In a focus plane, the amplitude of the twin-image depends on that of the real image, on the size of the holographed object and on the recording distance. Let $\vartheta$ be the object's aperture. In its focus plane, the reconstructed amplitude is given under a linear system approximation by the convolution with the system response:

$$
[\vartheta * R](x, y)=\vartheta(x, y)+2\left[\vartheta * \Re\left(\underline{h_{2 z}}\right)\right](x, y) .
$$

We derive in Appendix A an approximation of the twin-image for small and symmetrical objects (i.e. whose largest dimension $d$ verifies the condition $d \ll \sqrt{2 \lambda z}$ ):

$$
[\vartheta * R](x, y) \approx \vartheta(x, y)+2 \mathcal{F}\left[\vartheta_{i}\right]\left(\frac{x}{2 \lambda z}, \frac{y}{2 \lambda z}\right) \Re\left[\underline{h_{2 z}}(x, y)\right] .
$$

This approximation is useful to quantify the amplitude of the twin-image with respect to the real image. The twin-image approximate expression given in equation 4 involves the real part of Fresnel's function with parameter $2 z$ and the Fourier transform of the object's aperture scaled by a factor $1 /(2 \lambda z)$. The recording distance plays the role of a scaling factor: the twin-image is dilated when the recording distance increases. Then,

* Note that we do not consider here the effects due to sampling (pixel integration) and finite sensor size. In practice these affect the system response (i.e. perform a low-pass filtering). 
the larger the recording distance, the larger the spatial separation between the real image of the object and its superimposed twin-image.

The signal to noise ratio between the real image of an object and its corresponding twin image depends both on the recording distance and the object's size. For a given recording distance $z$, a dilation of the object by factor $a$ leads - according to the 2D Fourier transform scaling property - to a multiplication of the twin-image's amplitude by $a^{2}$. The wider the object, the higher the corresponding twin-image amplitude. Conversely, for a given object, the twin-image amplitude is proportional to the inverse of the recording distance $z$ through Fresnel's function. Hence, the larger the recording distance, the smaller the amplitude of the twin-image.

\section{Numerical suppression of the twin-image in the light of deconvolution techniques}

The many numerical techniques introduced in literature for twin-image suppression can be classified into distinct families of approaches. We will first briefly sum up these approaches and then show that there are strong connections between physicallybased, filtering and iterative approaches. Image deconvolution provides an interesting framework to give a unified description of these methods. On the basis of this description, we derive in section 3 an algorithm adapted to holograms of micro-objects spread in a volume.

\subsection{Classes of existing approaches}

Physically-based techniques: These techniques date back to the origins of holography. Bragg and Rogers[7] suggested the use of a second (auxiliary) hologram recorded at twice the distance of the main hologram to subtract the twin image. A similar approach can be conducted by subtracting from the reconstructed plane an estimate of the twinimage obtained by reconstructing an out-of-focus plane located at three times the infocus distance. The twin-image is then pushed back at twice its original distance and its amplitude reduced accordingly. This approach has been followed by authors using one $[6,8]$ or two $[9,10,11]$ holograms.

Inverse filtering approaches: Some authors[12, 13] try to invert the system response $R$ by designing an inverse filter in the Fourier domain. Different strategies are proposed to circumvent the filter singularities problem.

Iterative approaches: Iterative techniques have been designed[14, 15, 16] to improve the reconstructed amplitude, using a finite support constrain.

Phase retrieval techniques: With two or more holograms, more general phase reconstruction algorithms may also be used. Gerchberg-Saxton algorithm[17, 18] has 
been applied to digital holography in the work of Liu and Scott[19], and more recently in the work of Zhang et al. [20]. It iteratively reconstructs the phase in each hologram plane by performing back and forth propagation and enforcing at each step a constraint based on the measured hologram intensities. An improved version[21] of GerchbergSaxton algorithm has been suggested to account for the numerical errors $\left(\underline{h_{z}} * \underline{h_{-z}} \neq \delta\right.$ due to windowing effects).

Maximum a posteriori approach: Sotthivirat and Fessler suggested[22] a reconstruction of the image of a plane object using a maximum a posteriori approach (i.e. regularized image restoration). In the case of the study of small objects spread in a plane, the definition of an appropriate prior model is however difficult.

Other approaches: A quite different approach has been followed in [23]: the hologram is decomposed into blocks and reconstructed as a combination of off-axis sub-holograms. Tiller et al. suggest using two close holograms to deduce the phase on the basis of the connection between intensity deviation between two close planes and phase value.

\subsection{The deconvolution point of view}

Twin-image suppression has already been identified as a deconvolution problem by other authors (see [6]). We show here that the deconvolution provides a framework that reveals previously unnoticed connections between various approaches.

Let us consider a collection of $N$ objects defined by their apertures $\left(\vartheta_{i}\right)_{i=1 . . N}$. In a plane located at distance $z$ from the hologram, the complex amplitude due to the

objects is $\underline{a_{z}}=\sum_{i=1}^{N} \vartheta_{i} * \delta_{x_{i}, y_{i}} * h_{z_{i}-z}$, where $\left(x_{i}, y_{i}\right)$ is the transversal location of object $i$ and $z_{i}$ is the distance between object $i$ and the hologram plane. In the case of a single real-amplitude object, $a_{z}=\vartheta$ as is the case for objects spread in a unique plane located at the recording distance $z$. Note that phase objects such as micro-lenses or biological structures may also be considered, the aperture $\underline{\vartheta}$ then is complex-valued.

The intensity recorded in the hologram plane (after suppression of the continuous background constant) is given by [6]:

$$
I_{H}=-2 \Re\left(\underline{a_{z}} * \underline{h_{z}}\right)=-\underline{a_{z}} * \underline{h_{z}}-\operatorname{conj}\left(\underline{a_{z}}\right) * \underline{h_{-z}} .
$$

The numerically reconstructed amplitude in the plane $z$ is obtained[24] by convolving the hologram with Fresnel's kernel of parameter $-z$ :

$$
\underline{A_{R}}=I_{H} * \underline{h_{-z}}=-\underline{a_{z}}-\operatorname{conj}\left(\underline{a_{z}}\right) * \underline{h_{-2 z}}
$$

The common principle of all methods for numerical suppression of the twin-image is expressed either: (i) in the hologram plane (Eq. 5), or (ii) in the reconstructed plane (Eq. 6). 
2.2.1. Objects within a plane In this section, we consider that all objects are located at the same distance $z$ (the recording distance) from the hologram and that their transmittance $1-\vartheta_{i}$ is real-valued. The amplitude $a_{z}$ therefore is real and equations 5 and 6 become:

$$
\begin{aligned}
& I_{H}=-2 a_{z} * \Re\left(\underline{h_{-z}}\right), \quad \text { and } \\
& \underline{A_{R}}=-a_{z} *\left(\delta+\underline{h_{-2 z}}\right) .
\end{aligned}
$$

The twin-image suppression problem then corresponds to a deconvolution problem in both cases: (i) recover $a_{z}$ from $I_{H}$; and (ii) recover $a_{z}$ from $\underline{A_{R}}$. We describe in the following the main twin-image suppression techniques and underline the connections between each of them using the common framework of deconvolution techniques.

Fourier-quotient method: The basic technique to perform deconvolution is to design a filter in the Fourier domain that inverses the convolution kernel. The transfer function of the inverse filters corresponding to approaches (i) and (ii) are:

$$
\begin{aligned}
& \mathcal{F}\left[F_{I}^{(i)}\right](u, v)=\frac{1}{\cos \left[\lambda z \pi\left(u^{2}+v^{2}\right)\right]}, \quad \text { and } \\
& \mathcal{F}\left[F_{I}^{(i i)}\right](u, v)=\frac{1}{1+\exp \left[-2 \underline{j} \pi \lambda z\left(u^{2}+v^{2}\right)\right]} .
\end{aligned}
$$

These filters have a singularity for each zero of their denominator. Different solutions have been suggested:

- the transfer functions are set to one whenever a singularity is reached[12],

- a positive constant is added to the denominator[13],

- the filter is expanded into a series and only the first terms of the series are kept[6,8].

We give here the series expansion used in [8] as it will provide a direct connection with iterative approaches. It is based on the classical expansion $1 /(1+x)=1-x+\cdots+$ $(-1)^{n} x^{n}$ :

$$
\begin{aligned}
\mathcal{F}\left[F_{I}^{(i i)}\right](u, v) & =1-\exp \left[-2 \underline{j} \pi \lambda z\left(u^{2}+v^{2}\right)\right]+\exp \left[-4 \underline{j} \pi \lambda z\left(u^{2}+v^{2}\right)\right]-\cdots \\
& +(-1)^{n} \exp \left[-2 n \underline{j} \pi \lambda z\left(u^{2}+v^{2}\right)\right]
\end{aligned}
$$

and can be physically interpreted as pushing back the twin-image at distance $(2 n+2) z$ :

$$
\begin{aligned}
-\underline{A_{R}} * F_{I}^{(i i)} & =-\underline{A_{R}} *\left(\delta-\underline{h_{-2 z}}+\underline{h_{-4 z}}-\cdots+(-1)^{n} \underline{h_{-2 n z}}\right) \\
& =a_{z}+(-1)^{n} a_{z} * \underline{h_{-(2 n+2) z}} .
\end{aligned}
$$

Iterative approaches: The inverse filter $F_{I}^{(i i)}$ obtained by series expansion can be put into the following recursive form:

$$
\left\{\begin{array}{l}
\underline{a_{0}}=\underline{A_{R}} \\
\underline{a_{n+1}}=\underline{A_{R}}-\underline{a_{n}} * \underline{h_{-2 z}}
\end{array}\right.
$$


This corresponds exactly to the iterative algorithm of van Cittert[25, 26] applied to deconvolve equation 8 .

In the case of small objects, an a priori spatial constraint can be added if the support of the objects is known. Let us note $M$ as the binary function indicating whether point $(x, y)$ is in an object $(M(x, y)=1)$ or not $(M(x, y)=0)$. This spatial constraint can be enforced at each iteration (i.e. by projection on the sub-space of solutions that satisfy the constraint):

$$
\left\{\begin{array}{l}
\underline{a_{0}}=M \cdot \underline{A_{R}} \\
\underline{a_{n+1}}=M \cdot\left[\underline{A_{R}}-\underline{a_{n}} * \underline{h_{-2 z}}\right]
\end{array}\right.
$$

This algorithm corresponds to the one introduced by Lannes[14].

Koren et al. suggested[15, 16] another iterative algorithm also using a spatial constraint:

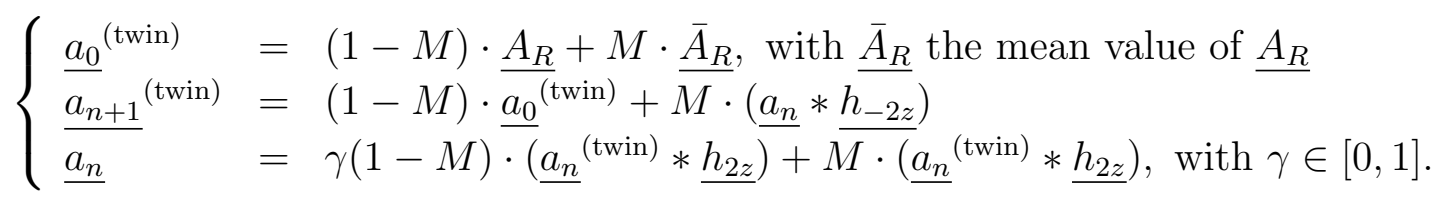

This algorithm goes back and forth between two planes corresponding respectively to the twin image $\underline{a}^{\text {(twin) }}$ and the real one $\underline{a}$. The underlying idea of this algorithm is that the real and virtual images carry the same information and can be interchanged through a convolution with $\underline{h_{2 z}}$ and $\underline{h_{-2 z}}$. Getting a real image without a spurious twin-image is indeed equivalent to getting a twin-image with no associated real image. The twin-image is well known outside the support of the real images. Inside this support, however, the twin and real images are superimposed. The algorithm therefore reconstructs iteratively the twin-image: at each step, the twin-image is updated inside the support $M$ and the real image is reduced outside $M$. This algorithm requires a good knowledge of the support $M$.

2.2.2. Objects in a volume When the studied objects are spread in a volume, the twinimage suppression problem expressed in equations 5 and 6 is no longer a deconvolution problem. However, if two holograms of the same objects - recorded at different distances or with a different wavelength — are available, they can be combined to obtain the classical deconvolution formulation. Let $I_{1}$ and $I_{2}$ be two holograms of the same objects respectively recorded at distances $z$ and $z+d$. Then, according to equation 5 , they are related to the complex amplitude of the objects through:

$$
-I_{1}+\left(I_{2} * \underline{h_{d}}\right)=\underline{a_{z}} * \underline{h_{z}} *\left(\delta-\underline{h_{2 d}}\right) .
$$

Several authors[12, 9, 10, 11] applied a deconvolution to equation 16 using similar approaches as those described as the Fourier-quotient methods in the previous paragraph.

Multi-hologram approaches (either deconvolution-based or performing phaseretrieval) are, however, limited in practice (except some specific setup like multiwavelength holography[27] or semi-transparent holograms in X-ray holography[28]) to 


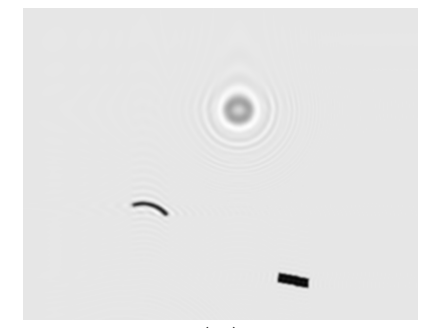

(a)

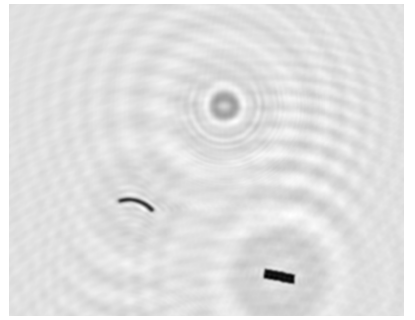

(b)

Figure 1. Twin-image appearing at the reconstruction step: (a) "twin-image free" reconstruction using the phase; (b) classical reconstruction from the hologram, showing the twin-image

the study of still objects as they require a recording of two holograms at different distances. We therefore suggest a new algorithm in section 3 that extends iterative approaches with a support constraint to the case of a single hologram of 3D objects.

\section{Algorithm for twin-image suppression in the case of 3D objects}

The use of a single hologram to reconstruct a twin-image free volume of objects prevents us formulating the problem directly as a deconvolution problem (see section 2.2.2). An iterative approach with finite-support constraint is however possible. It can then be interpreted in the context of wavelet denoising. Classical reconstruction gives images corrupted by the twin-images. Twin-image noise can be spatially localized by using a Fresnel transform with opposite parameter. The Fresnel transform is a redundant scaling transform[29] that separates, at some scales, signal (i.e. real images) from noise (i.e. twin-images). We can therefore apply the technique of wavelet coefficient thresholding[30].

In the following paragraphs we the algorithm that we suggest for twin-image suppression. Section 4 then illustrates some results on both simulated and real holograms.

\subsection{Principle}

The aim of the algorithm is to spatially separate the real and virtual images by masking. Figure 1 illustrates the twin-image phenomenon. Small diffracting objects were used to simulate the complex amplitude in a recording plane. The intensity of the field has been stored and two reconstructions have been performed. Image (a) has been computed from the simulated complex amplitude. It is therefore free of the twin-image phenomenon. Only two of the three objects are in focus. The third one is slightly out-of-focus. Image (b) is the result of the simulated hologram reconstruction. The lack of phase information results in the presence of the twin-image. Low frequency fringes appear in image (b) that could not be seen in image (a): this is the so-called "twin-image". In-focus images can easily be spatially separated from their twin-image. Out-of focus objects, on the other 
hand, cannot be separated. Twin-image suppression, in the case of objects in a volume, must be performed in 3D. In the following, we consider a set of planes $\left(z_{1}, \ldots, z_{p}\right)$ that coarsely sample the volume. The hard-thresholding wavelet coefficient algorithm can then be written:

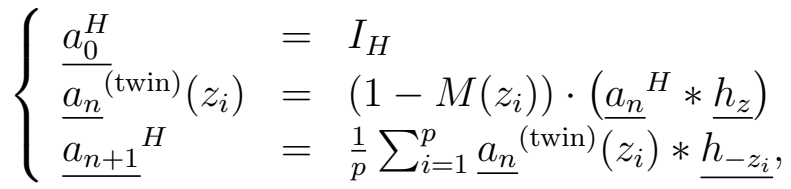

with $M$ being a 3D binary mask that indicates the support of the objects, and with $\arg (\underline{a})$ being the phase of complex number $\underline{a}$. We describe its construction in the next paragraph. The algorithm iteratively reconstructs the complex amplitude $\underline{a}^{H}$ in the hologram plane $I_{H}$. The noise is suppressed in each plane $\underline{a}^{(\text {twin) }}\left(z_{i}\right)$ of the twin halfspace.

The total redundancy of Fresnel transform is a drawback of the algorithm efficacy. If we consider the sampling of the volume into $p$ planes and that the twin-image of a given object is suppressed only in one of the $p$ planes by mask $M$, then the amplitude of this twin-image is reduced by factor $(p-1) / p$ after summing back all filtered planes. Direct use of the wavelet denoising procedure, therefore, is not usable. Instead of denoising the transformed planes, it is more efficient to suppress the phase corresponding to the twin-object in the hologram plane. This leads to the modified version of the algorithm illustrated on figure 2 , for $i=1$ to $p$ :

$$
\left\{\begin{array}{l}
{\underline{a_{0}}}^{H}=I_{H} \\
\left\{\begin{array}{l}
{\frac{a_{n_{(0)}}}{a_{n_{(i)}}}}^{H}=\underline{a}^{H} \\
\underline{a}_{n_{(i-1)}}^{H}-\left[M\left(z_{i}\right) \cdot\left({\underline{a_{n_{(i-1)}}}}^{H} * \underline{h_{z_{i}}}\right)\right] * \underline{h_{-z_{i}}} \\
{\underline{a_{n+1}}}^{H}={\underline{a_{n_{(p)}}}}^{H} .
\end{array}\right.
\end{array}\right.
$$

This time, the complex amplitude in the hologram plane $\underline{a}^{H}$ is modified $p$ times per iteration. The complex amplitude of the twin objects that are in focus in plane $z_{i}$ : $\left[M\left(z_{i}\right) \cdot\left({\underline{a_{n_{(i-1)}}}}^{H} * \underline{h_{z_{i}}}\right)\right] * \underline{h_{-z_{i}}}$ is subtracted from the hologram amplitude $\underline{a}^{H}$. The subtraction takes place before processing the next plane $z_{i+1}$ which avoids multiple suppression of a virtual object.

\subsection{Iterative cleaning}

The mask $M$ is obtained by rough segmentation of the real-objects half-space. In the case of amplitude-only objects, thresholding is sufficient to perform an approximate segmentation. Phase objects on the other hand might be segmented based on a focusing criteria such as that suggested by Dubois et al. [31] and on the phase in the reconstructed planes. Note however that we have not checked in this study the efficacy of the method on holograms in this latter case. 


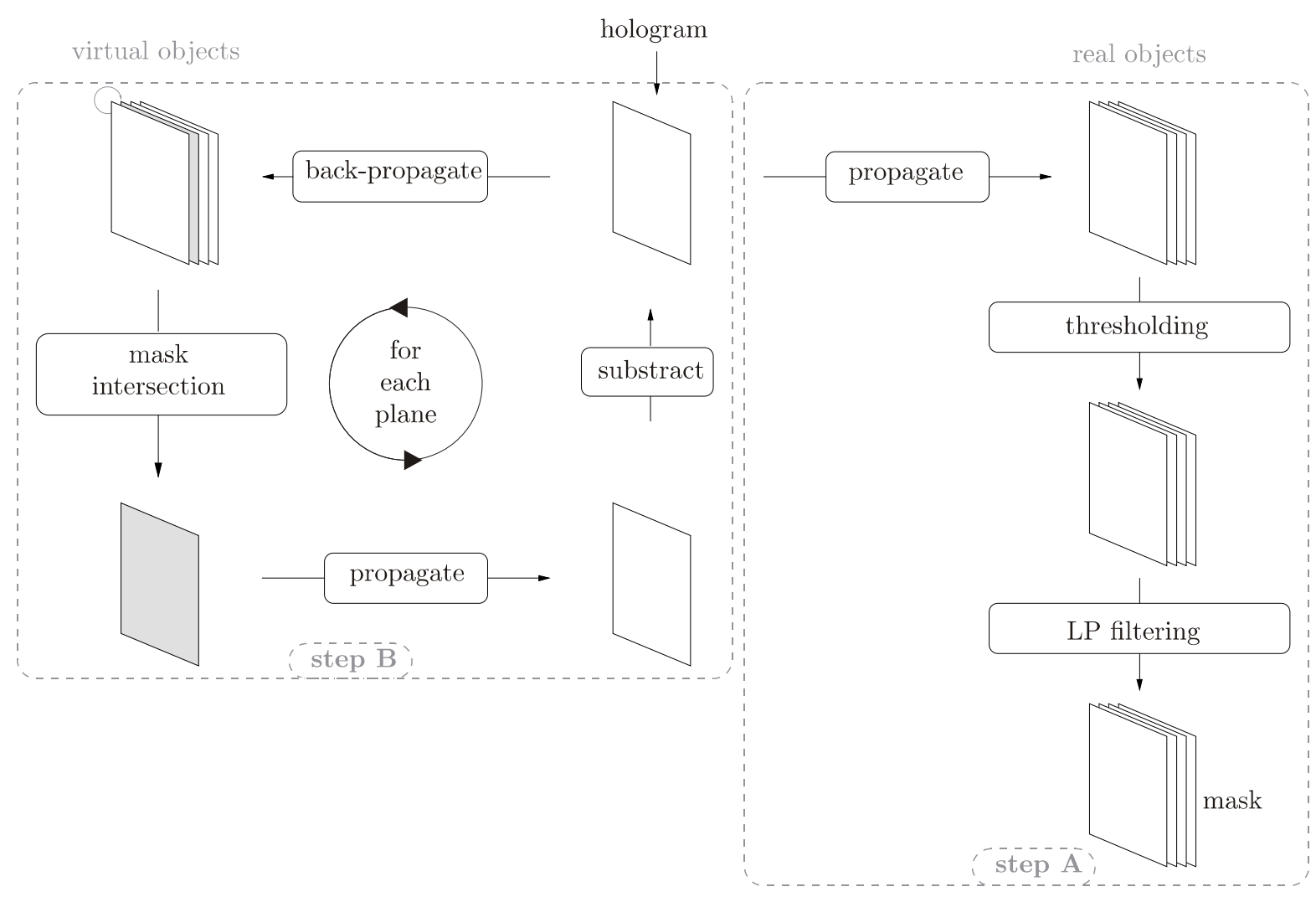

Figure 2. Twin-image cleaning scheme

The mask can be either computed once, before algorithm 18, or updated during the algorithm execution. In case of under-segmentation (i.e. non-detection of some objects) the twin-image suppression will be less efficient since the twin-images corresponding to the non-detected objects will remain.

To soften the constraints on the quality of the segmentation $M$ and to reduce the $z$ sampling steps, we suggest modifying the binary mask obtained by segmentation by: (i) dilating it with a disk a few pixels wide, (ii) low-pass filtering the result $(11 \times 11$ Gaussian kernel with 2 pixels standard deviation). It is then possible to suppress a twinimage even if the considered plane is not exactly in-focus. The low-pass filter is aimed at reducing sharp boundaries that could create ripples after Fresnel transform. The process of cleaning a twin-object from a slightly out-of-focus plane is illustrated in figure 3(a). A hologram of a disk-shaped object of $90 \mu \mathrm{m}$ diameter, recorded at $120 \mathrm{~mm}$ from the object, has been simulated. The cleaning plane is located at $2 \mathrm{~mm}$ from the focus-plane in order to prove that the $z$ sampling may be loose. The virtual object is efficiently suppressed in few iterations. The evolution of the relative energy of the virtual object is depicted in figure 3(b). The rapid decrease in the first iterations proves the efficiency of the cleaning algorithm. We found in practice that the twin-image suppression was satisfying after less than 10 iterations in the examples shown in section 4 . 


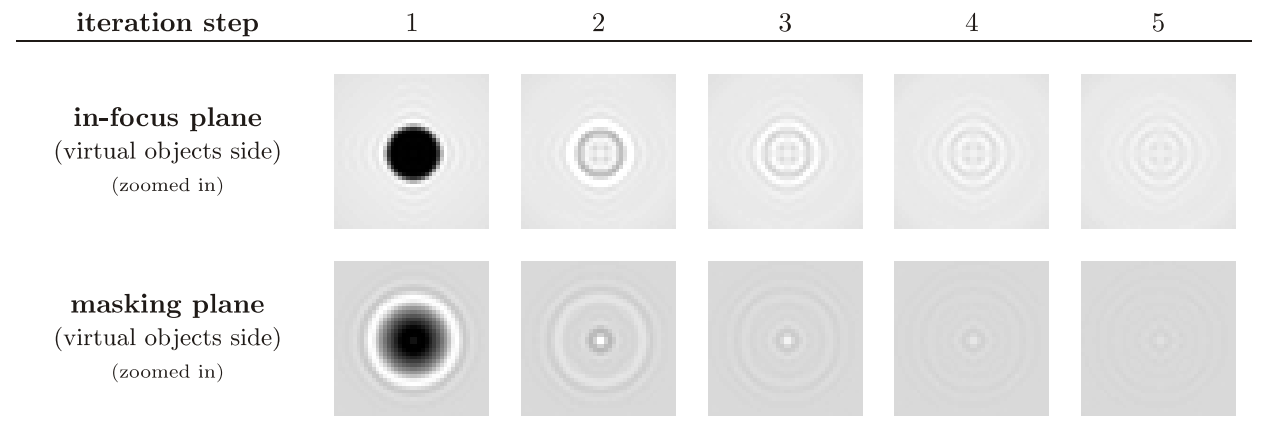

(a)

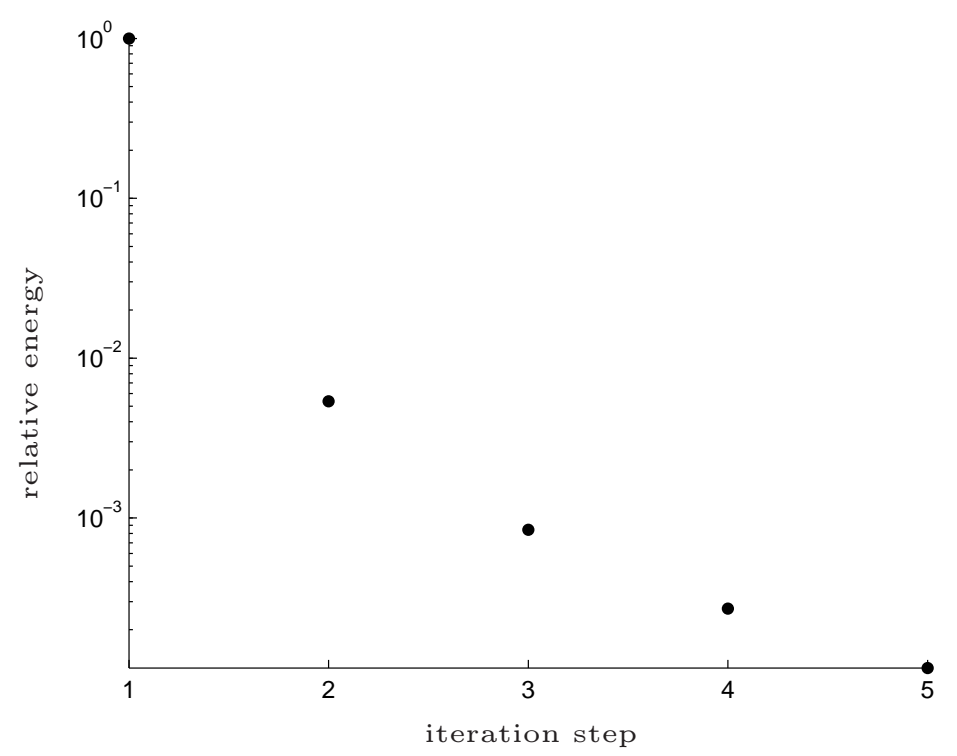

(b)

Figure 3. Illustration of the iterative suppression of a virtual object: (a) evolution of the twin-object in its in-focus plane (first row) and in the cleaning plane (second row) (please note that the contrast of the figure has been increased for visualization purposes); (b) relative energy of the twin-object at each iteration.

\section{Results}

We give some illustrations of the algorithm described in section 3 in three different cases: a simulated hologram of a large depth volume with some small objects; a simulated hologram of a uniform collection of particles; and an experimental hologram of water droplets.

Synthetic hologram of a simple three dimensional distribution of small objects The objects studied here are spread over three parallel planes each located at a $20 \mathrm{~mm}$ distance. The simulated hologram is recorded at $100 \mathrm{~mm}$ from the closest plane for a $1024 \times 1024$ sensor with $6.7 \times 6.7 \mu \mathrm{m}^{2}$ pixels $(8$ bit quantization depth). Figure $4(\mathrm{a})$ gives a diagram representing the object. The corresponding computed hologram is 
displayed in figure 4(b). Figures 4(c) to (e) show the three reconstructed planes using the classical reconstruction algorithm. The reconstructed planes after twin-image cleaning are given in figures $4(\mathrm{f})$ to $(\mathrm{h})$. The twin-image reduction is very efficient on this simple experiment.

Simulated hologram of opaque particles One hundred opaque spheres (diameter 90 $\mu m$ ) were spread in a $6 \times 6 \times 6 \times \mathrm{mm}^{3}$ volume. A hologram was numerically simulated at a distance $z=120 \mathrm{~mm}$ for a $1024 \times 1024$ sensor with $6.7 \times 6.7 \mu \mathrm{m}^{2}$ pixels (8 bit quantization depth). Figure 5(a) displays the hologram. Classically reconstructed and twin-image cleaned planes are displayed in figures 5(b) and 5(c) respectively. Five "cleaning" planes were used in the algorithm ( $p=5$ in algorithm 18). Figure 5(d) gives the signal to noise ratio in each 5 plane before (lowest curve) and after (highest curve) twin-image reduction. The signal to noise ratio have been computed by using the complex amplitude in the hologram plane as it is available in numerical simulations. Let us write $I_{R_{z}}^{(\text {th })}$ the theoretical intensity (i.e. the twin-image free computed with the complex amplitude) and $I_{R_{z}}^{(\mathrm{comp})}$ the intensity obtained with a reconstruction algorithm (i.e. classical reconstruction, or twin-image suppression using algorithm 18 prior to reconstruction). The signal to noise ratio shown on figure $5(\mathrm{~d})$ is:

$$
S N R=10 \log _{10}\left\{\frac{1}{\mathcal{N}} \sum_{x} \sum_{y}\left[\frac{I_{R_{z}}^{(\mathrm{th})}(x, y)}{I_{R_{z}}^{(\mathrm{th})}(x, y)-I_{R_{z}}^{(\mathrm{comp})}(x, y)}\right]^{2}\right\},
$$

with $\mathcal{N}$ the number of pixels. In this case, the twin-image reduction algorithm has increased the SNR by $10 \mathrm{~dB}$.

Experimental hologram of a water spray A cloud of water droplets was produced by a water spray. A hologram was recorded on a PCO Sensicam camera $(1280 \times 1024$ $6.7 \times 6.7 \mu m^{2}$ pixels with 12 bit quantization depth) using a YAG pulsed laser (lambda= $532 \mathrm{~nm}$, pulse duration $=7 \mathrm{~ns})$. The droplets were located within a bounding volume of $60 \mathrm{~mm}$ depth. Their size is roughly $90 \mu \mathrm{m}$. Figure 6(a) shows the experimental hologram. The classical reconstruction is given in figure $6(\mathrm{~b})$. The reconstruction after twin-image reduction is displayed in figure $6(\mathrm{c})$. The twin-image has been strongly attenuated. The algorithm seems robust against noise due to hologram acquisition (background noise due to spurious interference patterns). 


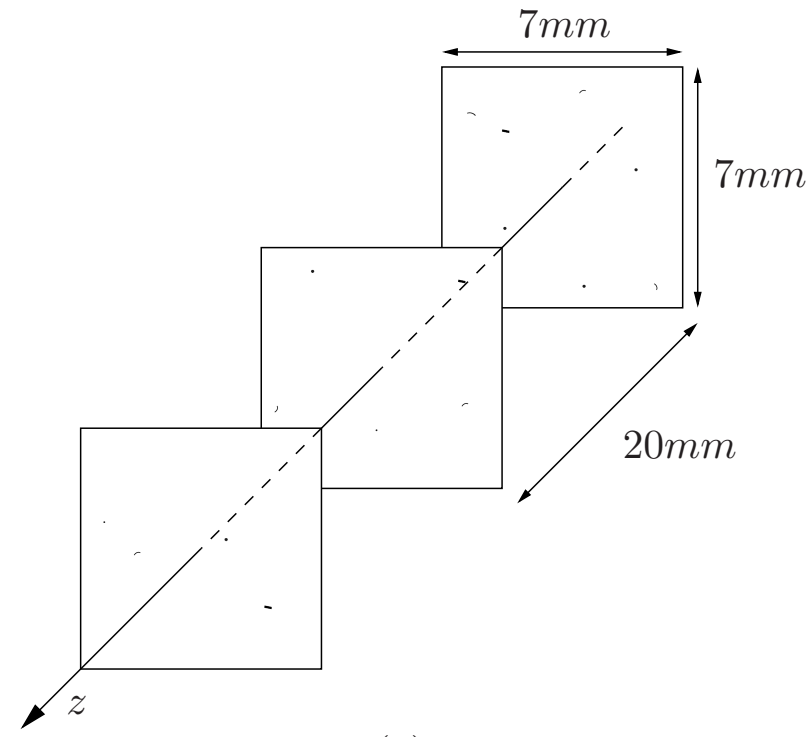

(a)

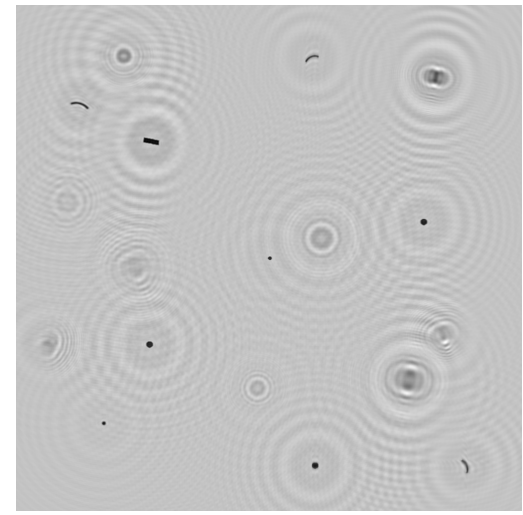

(c)

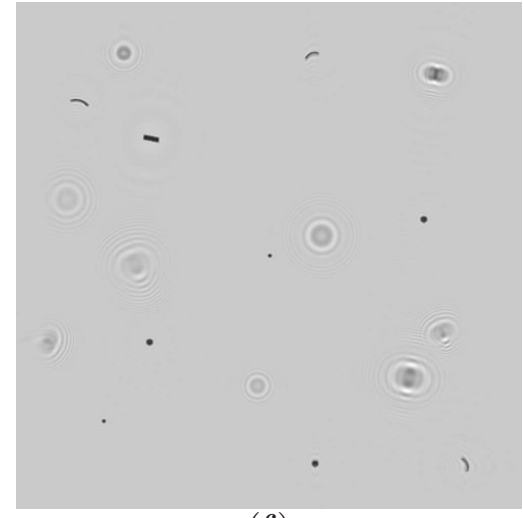

(f)

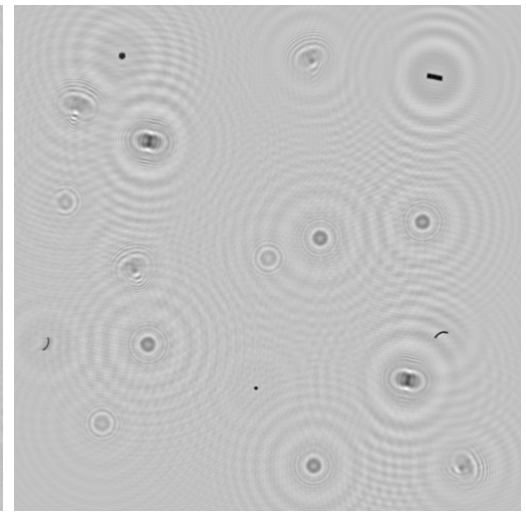

(d)

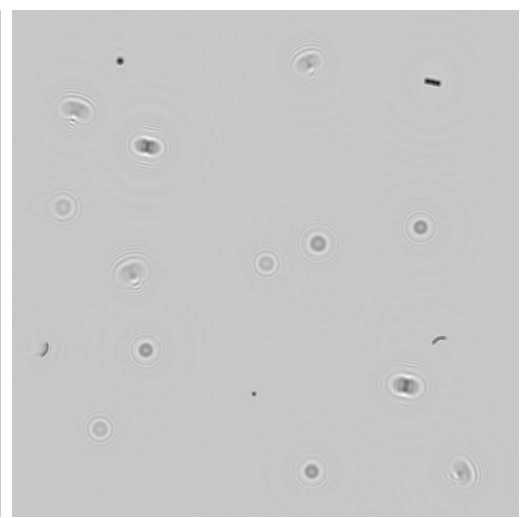

(g)

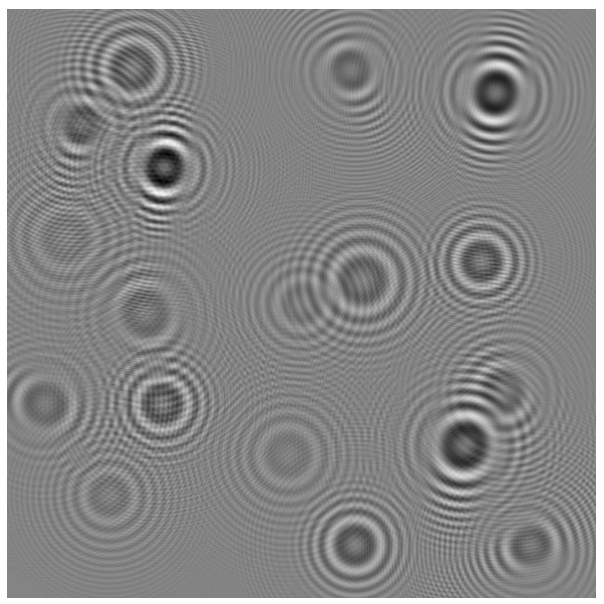

(b)

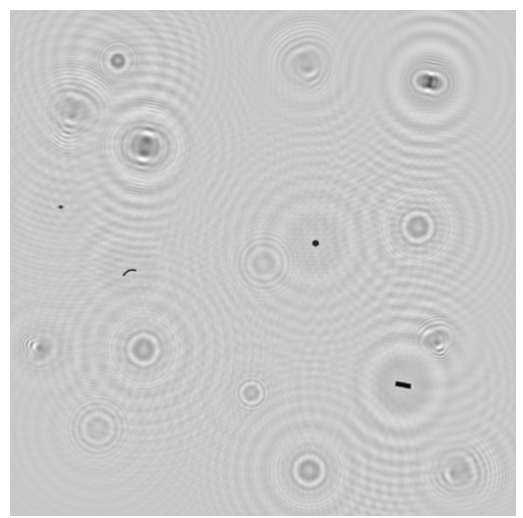

(e)

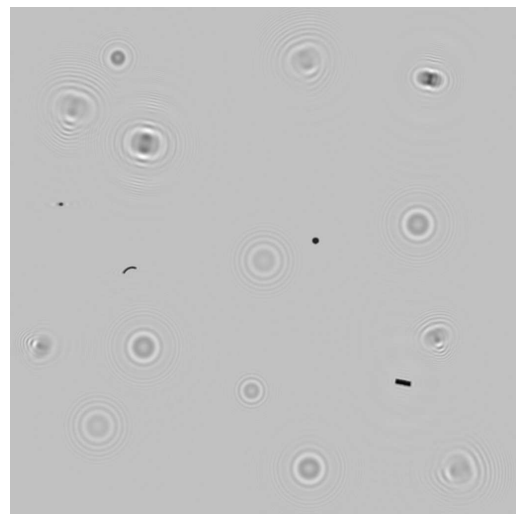

(h)

Figure 4. Twin-image cleaning applied on a simple synthetic hologram 


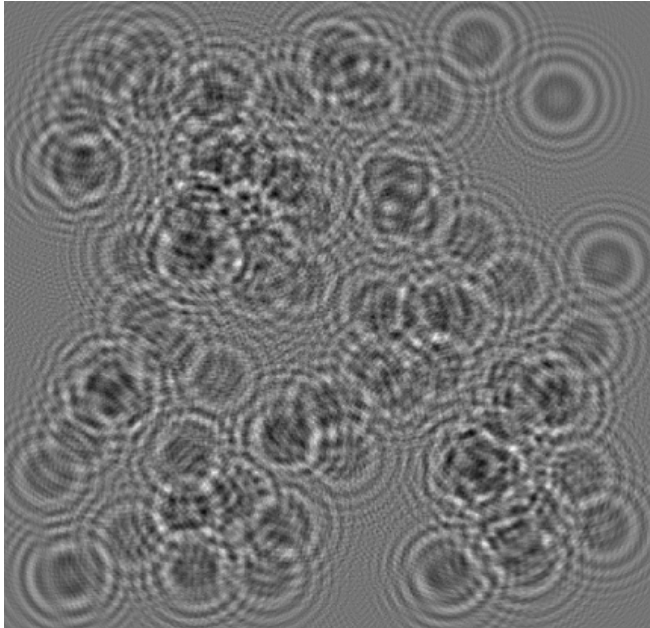

(a)

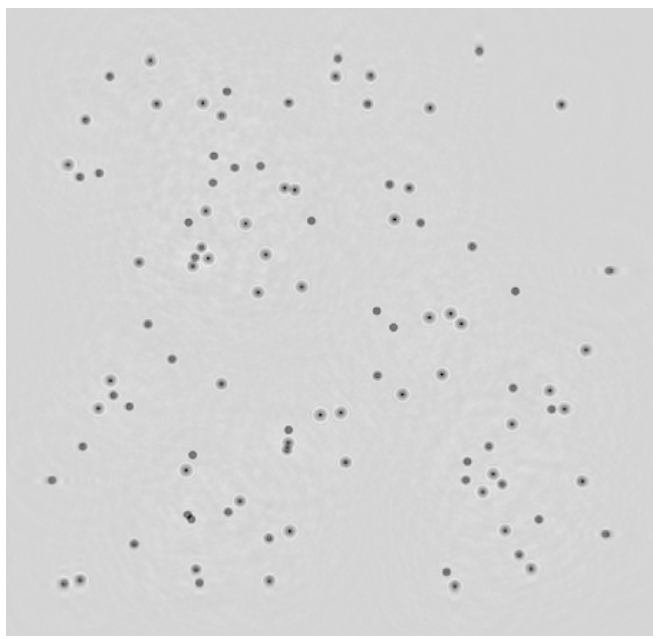

(c)

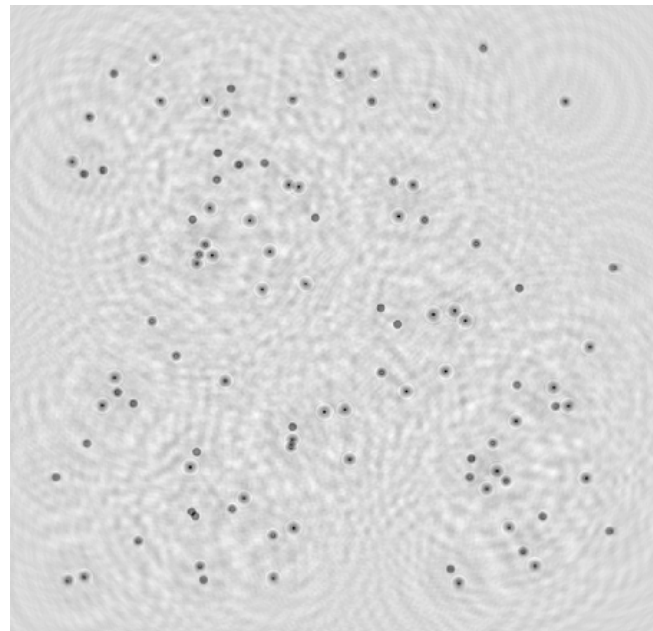

(b)

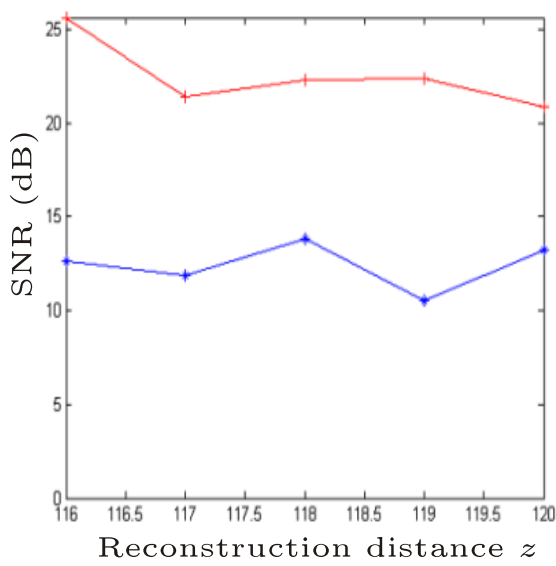

(d)

Figure 5. Twin-image cleaning applied on a synthetic hologram of 100 particles: (a) hologram; (b) a reconstructed plane; (c) the same plane after twin-image suppression; (d) comparison of the signal to noise ratio (SNR) for planes that sample the volume, the lower curve corresponds to classical reconstruction, the upper curve to twin-image suppression.

\section{Conclusion}

The twin image occurs because the traditionally used reconstruction does not invert the recording step. Numerical techniques for twin-image suppression try to improve the system response. We have shown that identifying the twin-image suppression problem as a deconvolution problem provides a unifying framework. We have set bridges between different methods previously described in the literature as being distinct approaches.

Existing algorithms cannot be applied to the case of small objects spread in a volume. We therefore derived an iterative algorithm that is both an evolution of finite- 


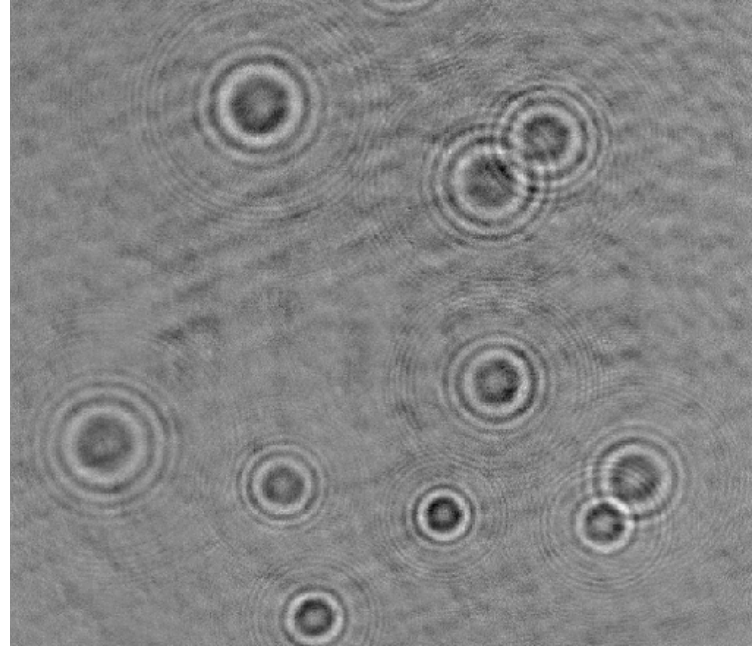

(a)

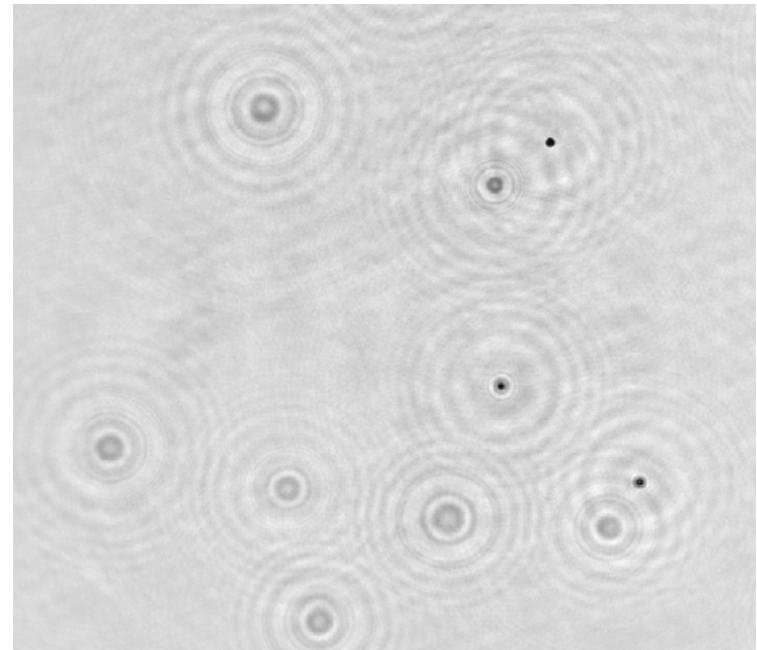

(b)

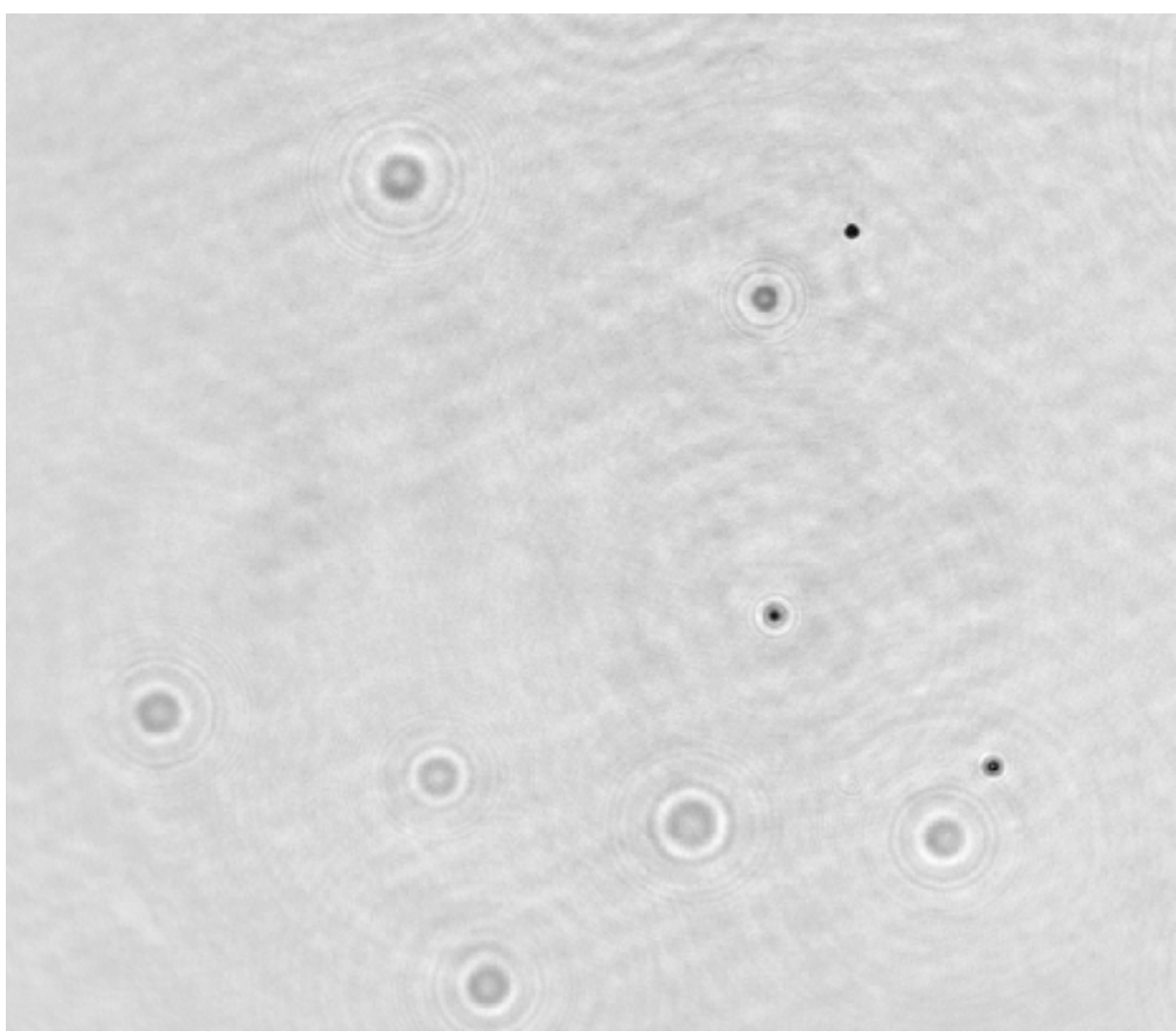

(c)

Figure 6. Twin-image cleaning applied on an experimental hologram of a water spray: (a) experimental hologram; (b) a reconstructed plane; (c) the same plane after twin-image suppression 
support techniques for plane objects and is inspired from wavelet denoising. The first results on simulated and real holograms are encouraging as the twin-images are efficiently cleaned. More generally, further experimental evaluation in various conditions should be completed to clarify the potential of the method.

It seems that the method could also be applied to holograms of phase-objects. An experimental validation on such holograms should however be considered.

The twin-image suppression technique we described might be usefull in particle holography for fluid dynamics. Its application for correlation-based measurements of particle holograms would suppress the indetermination due to the twin-image[32]. The algorithm could also be extended to twin and real objects suppression and possibly provide an alternative to Monnom et al. technique for out-of-focus object influence reduction[33]. It could then be used in applications such as interrogation cell correlation for velocity measurements[34] or multi-plane velocimetry[35]. Note that the described twin-image cleaning algorithm is limited to low or medium concentrated holograms as it relies on the spatial separation between real images and the surrounding twin-images. Furthermore, objects close to the hologram boundaries get blurred by the incorrect twin-image cleaning due to border effects. This problem requires further work and can be reduced by windowing[36].

Denoising and segmentation appear as tightly linked problems. For efficient twinimage suppression, denoising must be performed jointly with volume segmentation. The study of small objects in a volume (i.e. finite support objects surrounded by empty regions) suggests the use of adapted image processing techniques. Enforcing a finite support constraint as we do in this paper is a possible approach. In the case of well defined objects, such as spherical particles, a parametric estimation approach in an inverse problems framework proves to result in superior performance[37, 38].

\section{Appendix A. Approximate expression of the twin-image of a small object}

We derive here an approximate expression of the twin-image in the focus plane of the real image. We consider small objects, a situation common to particle holography and three dimensional imaging of small elements such as micro-organisms in water[39]. The twin-image amplitude is given (eq. 3) by:

$$
\begin{aligned}
{\left[\vartheta * \Re\left(\underline{h_{2 z}}\right)\right](x, y) } & =\Re\left[\vartheta *\left(\underline{h_{2 z}}\right)\right](x, y) \\
& =\Re\left[\frac{1}{2 \underline{j} \lambda z} \iint \vartheta(\xi, \eta) \exp \left(\underline{j} \frac{\pi}{2 \lambda z}\left[(x-\xi)^{2}+(y-\eta)^{2}\right]\right) \mathrm{d} \xi \mathrm{d} \eta\right],
\end{aligned}
$$

as we consider objects described by a real-valued aperture $\vartheta$.

This expression can be approximated by:

$$
\begin{aligned}
{\left[\vartheta * \Re\left(\underline{h_{2 z}}\right)\right](x, y) \approx \Re\left[\frac{1}{2 \underline{j} \lambda z} \exp \left(\underline{j} \frac{\pi}{2 \lambda z}\left[x^{2}+y^{2}\right]\right)\right.} \\
\\
\left.\qquad \int \vartheta_{i}(\xi, \eta) \exp \left(\frac{-\underline{j} \pi}{\lambda z}[x \xi+y \eta]\right) \mathrm{d} \xi \mathrm{d} \eta\right]
\end{aligned}
$$


for small objects such that $\frac{1}{2 \lambda z}\left[\xi^{2}+\eta^{2}\right]_{\max } \ll 1$, i.e. whose largest dimension $d$ verifies the condition $d \ll \sqrt{2 \lambda z}$.

The integration corresponds to the Fourier transform of $\vartheta$ up to a spatial scaling transform with a scale factor of $1 /(2 \lambda z)$ :

$$
\left[\vartheta * \Re\left(\underline{h_{2 z}}\right)\right](x, y) \approx \Re\left\{\underline{h_{2 z}}(x, y) \mathcal{F}\left[\vartheta_{i}\right]\left(\frac{x}{2 \lambda z}, \frac{y}{2 \lambda z}\right)\right\} .
$$

For symmetrical objects, the aperture $\vartheta$ is even and the Fourier transform is real. The twin-image is then given by:

$$
\left[\vartheta * \Re\left(\underline{h_{2 z}}\right)\right](x, y) \approx \Re\left\{\underline{h_{2 z}}(x, y)\right\} \mathcal{F}\left[\vartheta_{i}\right]\left(\frac{x}{2 \lambda z}, \frac{y}{2 \lambda z}\right),
$$

and appears clearly as a modulated signal in two ways:

- the frequency is linearly modulated with rate $r /(2 \lambda z)$, with $r$ the distance to the object center,

- the amplitude is modulated according to the Fourier transform of the object aperture.

\section{References}

[1] E.N. Leith and J. Upatnieks. Reconstructed wavefronts and communication theory. J. Opt. Soc. Am., 52(10):1123-1130, 1962.

[2] E. Cuche, P. Marquet, and C. Depeursinge. Spatial filtering for zero-order and twin image elimination in digital off-axis holography. Applied Optics, 39(23):4070-4075, 2000.

[3] L. Xu, J. Miao, and A. Asundi. Properties of digital holography based on in-line configuration. Optical Engineering, 39(12):3214-3219, 2000.

[4] I. Yamaguchi and T. Zhang. Phase-shifting digital holography. Optics Letters, 22(16):1268-1270, 1997.

[5] T.C. Poon, T. Kim, G. Indebetouw, B.W. Schilling, M.H. Wu, K. Shinoda, and Y. Suzuki. Twinimage elimination experiments for three-dimensional images in optical scanning holography. Optics Letters, 25(4):215-217, 2000.

[6] L. Onural and P.D. Scott. Digital decoding of in-line holograms. Optical Engineering, 26(11):11241132, 1987.

[7] WL Bragg and GL Rogers. Elimination of the unwanted image in diffraction microscopy. Nature, 167(4240):190-191, 1951.

[8] S. Yang, X. Xie, Y. Zhao, and C. Jia. Reconstruction of near-field in-line hologram. Optics Communications, 159:29-31, 1999.

[9] Y. Zhang and X. Zhang. Reconstruction of a complex object from two in-line holograms. Opt. Express, 11(6):572-578, 2003.

[10] Y. Zhang, G. Pedrini, W. Osten, and H.J. Tiziani. Reconstruction of in-line digital holograms from two intensity measurements. Optics Letters, 29(15):1787-1789, 2004.

[11] L. Denis, C. Fournier, T. Fournel, and C. Ducottet. Twin-image noise reduction by phase retrieval in in-line digital holography. In Wavelets XI, SPIE's Symposium on Optical Science and Technology, volume 5914, San Diego, CA, USA, 2005.

[12] M.H. Maleki and A.J. Devaney. Noniterative reconstruction of complex-valued objects from two intensity measurements. Optical Engineering, 33(10):3243-3253, 1994.

[13] K. A. Nugent. Twin-image elimination in Gabor holography. Optics Communications, 78:293-299, 1990.

[14] A. Lannes. Correction des effets de l'image jumelle et du terme quadratique en holographie de gabor. Optics Communications, 20(3):356-359, 1977. 
[15] G. Koren, D. Joyeux, and F. Polack. Twin-image elimination in in-line holography of finite-support complex objects. Optics Letters, 16(24):1979-1981, 1991.

[16] G. Koren, F. Polack, and D. Joyeux. Iterative algorithms for twin-image elimination in in-line holography using finite-support constraints. J. Opt. Soc. Am. A, 10(3):423-433, 1993.

[17] R.W. Gerchberg and W.O. Saxton. A practical algorithm for the determination of phase from image and diffraction plane pictures. Optik Vol, 35(2):237-246, 1972.

[18] J.R. Fienup. Phase retrieval algorithms : a comparison. Applied Optics, 21(15):2758-2769, 1982.

[19] G. Liu and P.D. Scott. Phase retrieval and twin-image elimination for in-line Fresnel holograms. J. Opt. Soc. Am. A, 4(1):159-165, 1987.

[20] Y. Zhang, G. Pedrini, W. Osten, and H.J. Tiziani. Whole optical wave field reconstruction from double or multi in-line holograms by phase retrieval algorithm. Optics Express, 11(24):3234-+, 2003.

[21] G. Yang, B. Dong, B. Gu, J. Zhuang, and O. Ersoy. Gerchberg-Saxton and Yang-Gu algorithms for phase retrieval in a nonunitary transform system : a comparison. Applied Optics, 33(2):209-218, 1994.

[22] S. Sotthivirat and J.A. Fessler. Penalized-likelihood image reconstruction for digital holography. J. Opt. Soc. Am. A, 21(5):737-750, 2004.

[23] S. Lai, B. Kemper, and G. von Bally. Off-axis reconstruction of in-line holograms for twin-image elimination. Optics Communications, 169:37-43, 1999.

[24] T.M. Kreis, M. Adams, and W. Juptner. Methods of digital holography : A comparison. In SPIE97, volume 3098, pages 224-233, Munich, Germany, 1997.

[25] P.H. van Cittert. Zum Einfluss der Spaltbreite auf die Intensitätverteilung in Spektrallinien. II. Zeitschrift für Physik A, 69(5-6):298-308, 1931.

[26] N.R. Hill and G.E. Ioup. Convergence of the van Cittert iterative method of deconvolution. J. Opt. Soc. Am., 66(5):487-489, 1976.

[27] B Javidi, P Ferraro, SH Hong, S De Nicola, A Finizio, D Alfieri, and G Pierattini. Threedimensional image fusion by use of multiwavelength digital holography. Optics Letters, 30(2):144-146, 2005.

[28] T Xiao, H Xu, Y Zhang, J Chen, and Z Xu. Digital image decoding for in-line x-ray holography using two holograms. Journal of Modern Optics, 45(2):343-354, 1998.

[29] L. Onural and M. Kocatepe. Family of scaling chirp functions, diffraction, and holography. IEEE transactions on signal processing, 43(7):1568-1578, 1995.

[30] H. Krim, D. Tucker, S. Mallat, and D. Donoho. On denoising and best signal representation. IEEE Transactions on Information Theory, 45(7):2225-2238, 1999.

[31] F. Dubois, C. Schockaert, N. Callens, and C. Yourassowsky. Focus plane detection criteria in digital holography microscopy by amplitude analysis. Opt. Express, 14(13):5895-5908, 2006.

[32] T. Ooms, W. Koek, J. Braat, and J. Westerweel. Exctracting 3d particle field displacement from on-axis digital holographic piv measurements. In Workshop on Digital Holographic Reconstruction and Tomography, Loughborough, England, 2007.

[33] O. Monnom, F. Dubois, C. Yourassowsky, and JC Legros. Improvement in visibility of an in-focus reconstructed image in digital holography by reduction of the influence of out-of-focus objects. Applied Optics, 44(18):3827-3832, 2005.

[34] L. Denis, T. Fournel, C. Fournier, and C. Ducottet. Cleaning digital holograms to investigate 3d particle fields. In 12th International Symposium on Flow Visualization, Göttingen, Germany, 2006.

[35] S. Cöetmellec, C. Buraga-Lefebvre, D. Lebrun, and C. Ozkul. Application of in-line digital holography to multiple plane velocimetry. Measurement Science and Technology, 12:1392-1397, 2001.

[36] E Cuche, P Marquet, and C Depeursinge. Aperture apodization using cubic spline interpolation: application in digital holographic microscopy. Optics Communications, 182(1-3):59-69, 2000.

[37] F. Soulez, L. Denis, C. Fournier, E. Thiébaut, and C. Goepfert. Inverse-problem approach for 
particle digital holography: accurate location based on local optimization. J. Opt. Soc. Am. A, 24(4):1164-1171, 2007.

[38] F. Soulez, L. Denis, E. Thiébaut, C. Fournier, and C. Goepfert. Inverse problem approach in particle digital holography: out-of-field particle detection made possible. J. Opt. Soc. Am. A, 24(12):3708-3716, 2007.

[39] E Malkiel, J Sheng, J Katz, and JR Strickler. The three-dimensional flow field generated by a feeding calanoid copepod measured using digital holography. Journal of Experimental Biology, 206(20):3657-3666, 2003. 